\title{
CLINICAL AND HISTOPATHOLOGICAL STUDY OF PRURITIC PAPULAR ERUPTIONS IN HIV PATIENTS AT A TERTIARY CARE CENTRE
}

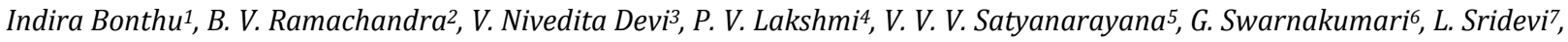 \\ T. P. Anilkumar ${ }^{8}$
}

\author{
${ }^{1}$ Assistant Professor, Department of DVL, Rangaraya Medical College, Kakinada. \\ ${ }^{2}$ Professor, Department of DVL, Rangaraya Medical College, Kakinada. \\ 3 Professor, Department of DVL, Rangaraya Medical College, Kakinada. \\ ${ }^{4}$ Associate Professor, Department of Pathology, Rangaraya Medical College, Kakinada. \\ ${ }^{5}$ Associate Professor, Department of DVL, Rangaraya Medical College, Kakinada. \\ ${ }^{6}$ Professor, Department of DVL, Rangaraya Medical College, Kakinada. \\ ${ }^{7}$ Assistant Professor, Department of DVL, Rangaraya Medical College, Kakinada. \\ ${ }^{8}$ ART Medical Officer, Rangaraya Medical College, Kakinada.
}

\begin{abstract}
\section{BACKGROUND}

Pruritic Papular Eruption (PPE) of HIV is the commonest cutaneous manifestation seen in HIV positive patients. PPE clinically presents as bilaterally symmetrical itchy papules over trunk and extremities especially in late stages of HIV infection posing a therapeutic challenge to the clinician for precise diagnosis and management.

Aims and Objectives- The present study was aimed to separate Pruritic papular eruption of HIV (PPE) which is an independent entity that clinically resembles other dermatoses presenting as pruritic papular lesions on the basis of clinical and histopathological data and to correlate with CD4 count.
\end{abstract}

\section{MATERIALS AND METHODS}

The prospective study is conducted in Department of Dermatology at Rangaraya Medical College, Kakinada, Andhra Pradesh. 78 clinically diagnosed cases of PPE were enrolled in the study after confirming HIV status and evaluated clinicohistopathological spectrum of PPE. CD4 count was done in all cases.

\section{RESULTS}

Out of 78 cases of PPE, 34 were male and 44 were female. 85\% of cases belong to 20-40 years age group. Heterosexual route is the major mode of transmission in 74 cases (94.9\%). Histopathological concordance was achieved in only 60 cases of PPE (76.92\%). Grade-1 only extremities in 5 cases (9\%), Grade-2 Trunk and extremities in 33 cases (55\%) and Grade-3 generalised in 22 cases (36\%). Histology showed hyperkeratosis, acanthosis in 47 cases (78.33\%), spongiosis in 38 cases (63.3\%) and perivascular, periadnexal, perifollicular infiltration of lymphocytes in all cases of PPE. CD4 counts less than 200 were found in 42 (71.66\%) PPE cases.

\section{CONCLUSION}

PPE of HIV is an independent entity distinguished from other conditions like eosinophilic folliculitis and papular urticaria by the involvement of perivascular, periadnexal and perifollicular infiltration of lymphocytes which is the characteristic feature. This work highlights the necessity of histopathology for proper diagnosis and management of PPE in HIV patients.

\section{KEYWORDS}

Pruritic Papular Eruption, HIV, Histopathology, CD4 Count.

HOW TO CITE THIS ARTICLE: Bonthu I, Ramachandra BV, Devi VN, et al. Clinical and histopathological study of pruritic papular eruptions in HIV patients at a tertiary care centre. J. Evolution Med. Dent. Sci. 2017;6(68):4842-4847, DOI: $10.14260 /$ Jemds/2017/1050

\section{BACKGROUND}

Dermatological complications of HIV can be a challenge to diagnose and manage. Pruritic papular eruption (PPE of HIV) is a common cutaneous manifestation of HIV causing significant morbidity in HIV positive patients. The prevalence of PPE in HIV positive patients varies between 11 to $46 \%$

Financial or Other, Competing Interest: None.

Submission 20-07-2017, Peer Review 13-08-2017,

Acceptance 19-08-2017, Published 24-08-2017.

Corresponding Author:

Indira Bonthu,

Assistant Professor,

Department of DVL,

Rangaraya Medical College, Kakinada.

E-mail:nallaindira4554@gmail.com

DOI: $10.14260 /$ jemds $/ 2017 / 1050$ depending on the geographic area.(1) PPE is characterised by chronic pruritus and symmetric papular eruption on the trunk and extremities in the absence of other definable causes of pruritus in a HIV infected patient.(2) The trunk and extremities are affected more than the face with sparing of mucous membranes, palms and web spaces. PPE is a skin sign of advance HIV infection being three times more common when the CD4 count is less than 200 cells per microlitre.(3)

Several chronically itchy papular and papulopustular eruptions variously referred to as PPE of HIV infection, itchy red bump disease, eosinophilic pustular folliculitis, eosinophilic folliculitis, papular urticaria, prurigo nodularis, insect bite reactions have been reported in HIV infected patients.(4) It is necessary to consider and exclude other conditions before a diagnosis of PPE was made. Common papular eruptions specific to HIV individuals include PPE and 
Eosinophilic folliculitis.(5) Clinically they are difficult to distinguish from each other. Histopathological examination may help to differentiate PPE from eosinophilic folliculitis especially to evaluate the involvement of follicle that is spared in PPE.(6)

PPE may be considered as a surrogate marker of immunosuppression and an indicator for the need to initiate Antiretroviral therapy in resource-poor settings where CD4 count facilities are not available.(7) The existing literature on PPE in Indian studies is scant.

Hence, we have made an attempt to separate these closely related entities by analysing the clinical and histopathological data and to correlate with CD4 count.

\section{MATERIALS AND METHODS}

Among 101 untreated cases of HIV a total of 78 HIV positive Patients with clinical characteristics of PPE attending Dermatology OPD at Rangaraya Medical College, Kakinada between October 2006 and April 2008 were selected for the study. HIV status was confirmed by performing ELISA/Rapid/simple tests. The study was approved by institutional ethics committee.

\section{Inclusion Criteria}

HIV positive Patients attending Department of Dermatology clinically presenting with pruritic papular lesions on the trunk and extremities of more than one month duration were included in the study.

\section{Exclusion Criteria}

Patients on ART, cutaneous infection or infestation, drug reaction, systemic complications, pregnant women, children less than 12 years of age were excluded from the study.

\section{Study Design}

This study is a descriptive study to differentiate PPE of HIV from papular eruptions in HIV positive patients and to correlate with CD4 count. PPE of HIV means cases presenting with bilaterally symmetric papular eruption on trunk and extremities of more than one month duration confirmed histopathologically. Participants of the study $n=78$ cases. Written informed consent was taken from all patients. All study participants were examined by study dermatologists for confirmation. Details regarding onset, duration, distribution of lesions and secondary changes were noted. The severity of rash was evaluated by rash severity scale.

Grading of PPE was done based on the site of involvement. Grade 1 (mild) is involvement of extremities, grade 2 (moderate) is involvement of trunk and extremities and grade 3 (severe) is generalised (including face) involvement.

During the cutaneous examination patients were asked to identify the recent onset lesions and were selected for Biopsy. After giving local anaesthesia a 4-mm punch was taken. Tissue samples were formalin fixed and sent for histopathological examination. CD4 counts were done for all cases by flow cytometry method. Clinical diagnosis was supplemented with laboratory procedures like microscopy, $\mathrm{KOH}$ preparation where ever needed.

\section{Statistical Analysis}

All the data were analysed by use of non-parametric analysis by the variance and chi square test. The Mann-Whitney test was used to compare non-normally distributed values such as CD4 cell count.

\section{RESULTS}

Among 101 untreated cases of HIV, 78 patients were completely evaluated. Out of 78 patients, 34 were male, 44 were female with a male to female ratio of 1:1.4. Although there is no statistically significant difference between age groups and gender (Fisher exact test p value being 1) implying that these two variables were independent. (See table 1) females presented early probably due to the stigma. Majority of the cases (85\%) belong to the age group 20-40 years. In 74 cases $(94.9 \%)$, heterosexual route is the major mode of transmission. out of 78 cases ( $\mathrm{n}=78$ cases) histopathologically confirmed cases were Pruritic papular eruption (PPE) of HIV 60 cases (76.92\%). Eosinophilic folliculitis 8 cases (10.25\%) Papular urticaria 4 cases (5.13\%), Pityrosporum folliculitis 4 cases $(5.13 \%)$, prurigo simplex 1 case $(1.26 \%)$ and pemphigus foliaceus in 1 case (1.26\%). (See Table 2).

The mean age of the patients is 32.5 years (ranging from $20-55$ years), the mean duration of PPE is 9.5 months. Mean duration of HIV positivity is 28 months. Duration from HIV positive to onset of PPE is 24.2 months.

Grading of PPE was done based on the involvement of trunk and extremities. Grade 1 (mild) is observed in $5(9 \%)$ cases. Grade - 2 (moderate) in 33 cases (55\%) and Grade -3 (severe) in 22 cases (36\%) (See Table 2). PPE lesions were healed with Hyperpigmentation in 21 cases (35\%) and healing with scarring was seen in 39 cases (65\%). By using H\&E staining, we have observed significant differences in the infiltrate. Presence of lymphocytic infiltrate is seen in PPE of HIV. Presence of perifollicular infiltrate of eosinophils is seen in Eosinophilic folliculitis of HIV. Presence of neutrophilic infiltrate is observed in Pityrosporum folliculitis.

Biopsy features showed epidermal changes that include Hyperkeratosis in 47 cases (78.33\%), Parakeratosis in 44 cases (73.3\%), Focal acanthosis in 48 cases (80\%), Spongiosis in 38 cases (63.3), Flattening of rete ridges in 36 cases $(60 \%)$, Necrotic Keratinocytes in 23 cases (3\%), Erosion of epidermis in 6 cases $(10 \%)$, Subepidermal bulla in 16 cases (26.6\%). Perivascular, periadnexal and perifollicular infiltrates in the dermis is observed in all cases of PPE (see Table 3).

In 42 cases of PPE (71.66\%), CD4 count was less than 200 indicating that it is an advanced sign of HIV. (See Table 4). In 7 out of 8 cases of eosinophilic folliculitis, CD 4 count was less than 250. In all 4 cases of Pityrosporum folliculitis, CD4 count was more than 350 indicating that it is an early sign of immunosuppression.

\begin{tabular}{|c|c|c|c|c|c|}
\hline $\begin{array}{c}\text { Age } \\
\text { (Years) }\end{array}$ & Male & Female & Total & $\mathbf{\%}$ & $\begin{array}{c}\text { Fisher } \\
\text { exact test } \\
\text { P value }\end{array}$ \\
\hline $18-40$ & 27 & 34 & 61 & 43.59 & \multirow{2}{*}{1 (NS) } \\
\cline { 1 - 5 } $40-60$ & 7 & 10 & 17 & 56.41 & \\
\hline Total & $\mathbf{3 4}$ & $\mathbf{4 4}$ & $\mathbf{7 8}$ & $\mathbf{1 0 0 . 0}$ & \\
\hline \multicolumn{7}{|c|}{ Table 1. Age-sex Distribution } \\
\hline
\end{tabular}




\begin{tabular}{|c|c|}
\hline Pruritic Papular eruptions & 60 \\
\hline Eosinophilic Folliculitis & 8 \\
\hline Papular Urticaria & 4 \\
\hline Pityrosporum Folliculitis & 4 \\
\hline Prurigo Simplex & 1 \\
\hline Pemphigus Foliaceus & 1 \\
\hline Table 2. Results
\end{tabular}

\begin{tabular}{|c|c|c|c|c|c|c|}
\hline Site of Involvement & PPE & EF & PU & PF & PS & PE. F \\
\hline Grade 1 (mild) & $5(9 \%)$ & 2 & & & & \\
\hline Grade 2 (moderate) & $33(55 \%)$ & 6 & 4 & 4 & 1 & 1 \\
\hline Grade 3 (severe) & $22(36 \%)$ & & & & & \\
\hline $\begin{array}{c}\text { Healing with } \\
\text { hyperpigmentation }\end{array}$ & $21(35 \%)$ & & & & & \\
\hline Healing with scarring & $39(65 \%)$ & & & & & \\
\hline Table 3. Grading of PPE \\
\hline
\end{tabular}

\begin{tabular}{|c|c|}
\hline Epidermal Changes & Cases \% \\
\hline Hyperkeratosis & $47(78.33 \%)$ \\
\hline Parakeratosis & $44(73.3 \%)$ \\
\hline Focal acanthosis & $48(80 \%)$ \\
\hline Spongiosis & $38(63.3 \%)$ \\
\hline Flattening of rete ridges & $36(60 \%)$ \\
\hline Necrotic keratinocytes & $23(38.3 \%)$ \\
\hline Erosion & $6(10 \%)$ \\
\hline Subepidermal bulla & $16(26.6 \%)$ \\
\hline \multicolumn{2}{|c|}{ Table 4. Histopathological Findings } \\
\hline
\end{tabular}

\begin{tabular}{|c|c|c|c|}
\hline CD4 Count & PPE Cases & Non PPE Cases & Row Totals \\
\hline$<50$ & $4(5.45)[0.39]$ & $3(1.55)[1.37]$ & 7 \\
\hline $50-100$ & $12(13.25)[0.12]$ & $5(3.75)[0.41]$ & 17 \\
\hline $100-200$ & $27(24.16)[0.33]$ & $4(6.84)[1.18]$ & 31 \\
\hline $200-300$ & $11(9.35)[0.29]$ & $1(2.65)[1.03]$ & 12 \\
\hline$>300$ & $6(7.79)[0.41]$ & $4(2.21)[1.45]$ & 10 \\
\hline $\begin{array}{c}\text { Column } \\
\text { Totals }\end{array}$ & $\mathbf{1 8}$ & $\begin{array}{c}\mathbf{7 8} \text { (Grand } \\
\text { Total) }\end{array}$ \\
\hline \multicolumn{3}{|c|}{ Table 5. Correlation of PPE with CD4 Count } \\
\hline
\end{tabular}

The chi square statistic is 6.9899 . The $\mathrm{p}$ value is 0.136421 . (Not significant).

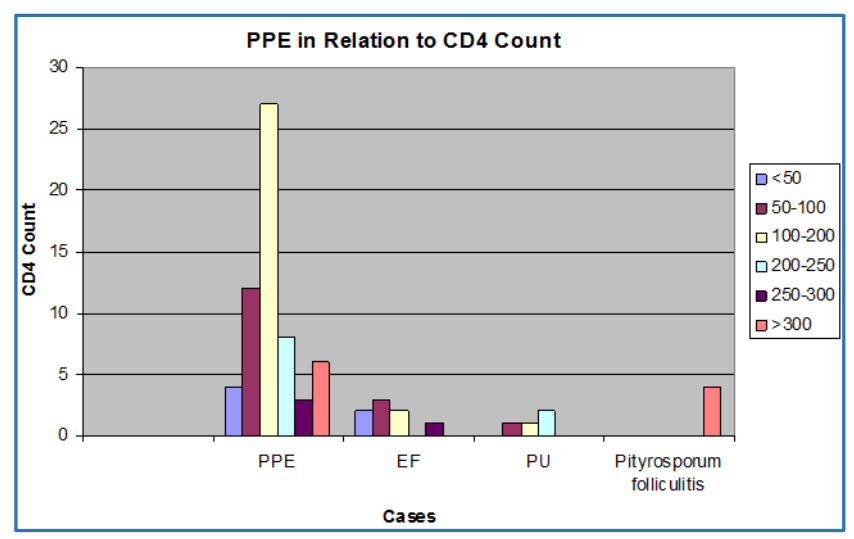

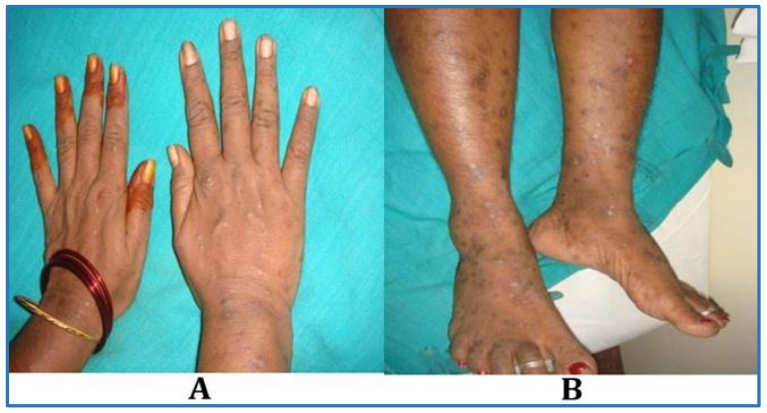

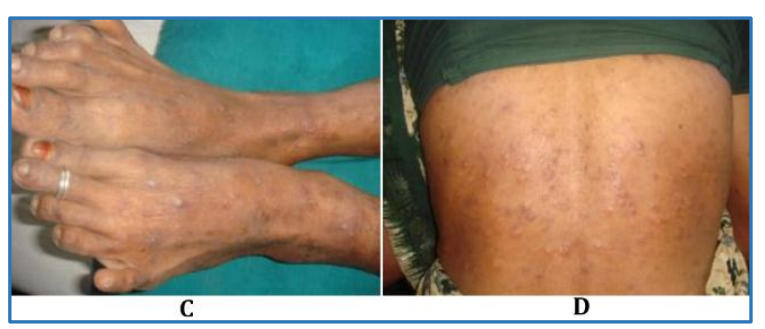

Figures $1 A, B, C$, $D$ showing Distribution of PPE (A) (B) (C) and (D)

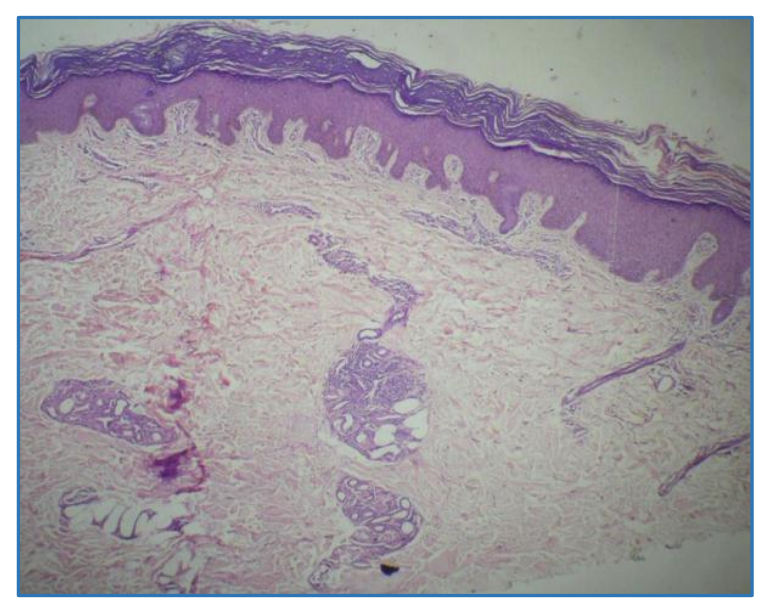

Figure 2A. Showing Hyperkeratosis, Acanthosis, Periadnexal Infiltration of Lymphocytes (10x) H\&E Stain

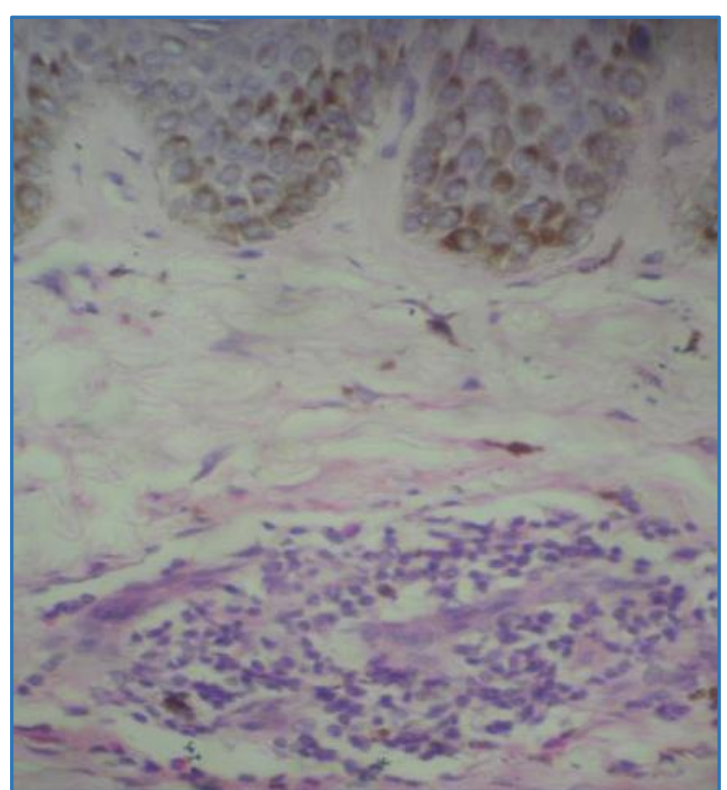

Figure 2B. Showing (High power 40x) Spongiosis and Lymphocytes 


\section{DISCUSSION}

Cutaneous findings in HIV infection are not unique and may resemble other infectious and non-infectious diseases. The frequency distribution of common cutaneous manifestations varied among several studies but the most common finding was PPE. Though initially in early 1990s, PPE cases were reported in African countries but now it is more prevalent in south Asian countries particularly in tropical areas. $22.5 \%$ of newly diagnosed HIV-positive patients visiting a tertiary care centre were found to have PPE in an Indian study. ${ }^{(8)}$

In the present study, a total of 78 HIV cases with characteristics of PPE were studied. Out of 78 cases, 60 cases were histologically confirmed as PPE of HIV (76.92\%) which is similar to $79.2 \%$ of Terry Farconi et al study at Chennai in 2008. According to recent statistics, the incidence of HIV in women is increasing dramatically and now nearly half of the 40 million cases of HIV/AIDS worldwide were women.(9) In India, adult HIV prevalence (15-49) years is $0.3 \%$ among males and $0.22 \%$ among females.(10) In the present study, 44 cases were female with a male to female ratio of $1: 1.4$ and $60 \%$ were illiterates. Most common age group is $20-40$ years. This increased incidence in females and younger individuals is probably due to early marriages, involving in sexual activity at an early age, illiteracy, poverty and malnourishment. National HIV prevalence is $0.36 \%$ with maximum number of infections in high risk population like commercial sex workers, men having sex with men and intravenous drug abuse. (11)

Heterosexual route is the major mode of transmission of HIV in 74 cases (94.9\%). There is history of casual contact with friend/relative/unknown persons observed in 47 cases $(60.26 \%)$ followed by contact with commercial sex worker (38.46\%) indicating that there is need for health education and proper counselling to reduce their high-risk behaviour. Previous studies have not found any significant differences in patients living in rural versus urban areas in their risk for PPE. In the present study, more than $90 \%$ of cases belong to rural areas wherein ditches and ponds were present in large numbers. Majority of the cases were labourers (56\%) who work in agriculture fields. These findings suggest that probably there is role of exogenous agent (mosquito bites) in causing PPE.(12)

Colebunders et al observed that $51 \%$ of HIV-positive patients had generalised PPE of unknown aetiology of at least 1 month duration as their initial disease manifestation. Also, 95\% patients with PPE and severe weight loss (greater than $10 \%$ of normal body weight) were seropositive.(13) PPE can be the first marker of HIV infection. (14) In the present study, 21 cases $(35 \%)$ of PPE presented as initial manifestation in HIV patients. The mean duration of PPE is 9.5 months and mean duration of HIV positivity is 28 months and duration from HIV positive to the onset of PPE is 24.2 months. In previous studies, it was reported that PPE is a sign of advanced HIV,(15) but these results indicate that PPE is often an early sign of HIV and in majority of cases patients developed PPE within 2 years of HIV seropositivity. Thus, recognising PPE early in the course of HIV helps in early diagnosis and treatment.

Resneck and colleagues believe that PPE is indeed a reaction to arthropod bites which is supported by several authors as a shift from Th1 to Th2 cytokine profile. Patients who have Th2 dominance may be more predisposed to the development of arthropod hypersensitivity leading to PPE.(16)
The severity of PPE was evaluated by asking two questions whether the lesions were itchy for the past one month or not and whether the lesions were interfering with sleep or not.(17) Using the rash severity scale, the severity of skin rash was not significantly associated with the CD4 count. This result is in contrast with the previous studies and implies that we can use neither rash distribution nor severity as an indicator of CD4 count.

In the present study, grade 1 involvement of extremities was observed in only $9 \%$ of cases. In most of the cases initially only extremities are involved and later becoming generalised. Diffuse distribution of lesions can further explained by the ability of arthropod bites including mosquito bites through clothing and as the CD4 counts decline patients with PPE may become hypersensitive to arthropod antigens that they previously may have tolerated. Majority of patients with PPE had severe pruritus resulting in excoriations which is due to chronic recall reaction to the arthropod antigens.(18) Most patients scratch because of severe pruritus resulting in post inflammatory hyperpigmentation in 21 cases $35 \%$ and scar formation in 39 (65\%) of cases. In the present study, $90 \%$ of the patients had severe itching.

In the present study, biopsy findings in the epidermis showed hyperkeratosis, acanthosis and spongiosis as the predominant features similar to arthropod bite reactions were observed which is on par with Uganda study. Trafficking of lymphocytes occur continuously in the epidermis as a part of immune surveillance. There can be no spongiosis without lymphocytes in the epidermis to initiate the reaction.

In the present study, superficial perivascular, periadnexal, perifollicular infiltrate of lymphocytes was seen in all cases $(100 \%)$ which is the characteristic feature of PPE of HIV Neutrophil infiltrate is seen in $36(60 \%)$ cases, eosinophilic infiltrate in $12(20 \%)$ cases.

Most of the patients had other skin diseases or opportunistic infections before the development of PPE.(19) At the time of clinical examination, some cutaneous findings were noted. The commonest association is onychomycosis in 26 cases $(40.33 \%)$ followed by diffuse thinning of hair in 15 cases $(25 \%)$, oral candidiasis in 10 cases $(16.66 \%)$ furunculosis in 6 cases $(10 \%)$ herpes zoster in 6 cases $(10 \%)$, genital herpes in 4 cases $(6.66 \%)$ herpes simplex labialis in 2 cases $(3.33 \%)$ molluscum contagiosum in 2 cases $(3.33 \%)$ cutaneous fibrous histiocytoma in 2 cases $(3.33 \%)$, plane warts in 1 case (1.66\%). HIV associated Eosinophilic folliculitis predominantly involves face and upper extremities rather than lower half of the body.(20) Our study supports this finding as 6 out of 8 cases there is face and trunk involvement. on histology, perifollicular infiltrate of eosinophils with CD4 count less than 250 and in Pityrosporum folliculitis multiple discrete, perifollicular papules involving trunk, proximal arms, neck showing histological features of follicular plugging, dilated adnexal ducts and neutrophilic infiltrate forming abscess formation in epidermis with CD4 more than 350. Though in our study we got less number of cases the activity of Pityrosporum in HIV is very well known. Pityrosporum ovale, is a part of normal skin flora, extensively colonises, due to the changes in immunity in presence of HIV produces cell mediated response resulting in inflammation.(21) In all 4 cases $(100 \%)$ 
of Pityrosporum folliculitis had CD4 count more than 350 indicating that it is an early manifestation of HIV.

Papular urticaria or arthropod bite reactions may be indistinguishable clinically from PPE and can be excluded by a history of pets at home, seasonal variation, or location of lesions ${ }^{(22)}$ in the present study 4 cases of papular urticaria had CD4 count less than 250. Though in previous studies it was stated that lower levels of IL2 and gamma IFN and negative correlation between gamma IFN and peripheral CD4 lymphocytes may indicate an early phase of immunosuppression in $\operatorname{PPE}^{(23)}$ but now it is considered as advanced sign of HIV Infection where majority of patients with PPE had CD4 count less than 200.(24) Several observational studies have reported that there is a link between low CD4 count and advanced HIV disease and as per WHO clinical staging it is included in stage III and IV and thus proposing PPE as a marker for advanced HIV.(25) Sanchez et al found that $80 \%$ had CD4 counts below 100.(26)

In the present study, out of 60 cases of PPE, 43 cases had CD4 less than 200, 13 cases had CD4 between 200-350 and 4 cases had CD4 more than 350. On initiation of HAART therapy, PPE lesions were cleared in most of the cases. Out of 8 cases of eosinophilic folliculitis, 4 cases had CD 4 less than 100 and 4 cases had CD4 between 100-250.

\section{CONCLUSION}

Pruritic papular eruption of HIV is an independent entity characterised clinically by intense pruritus, diffuse distribution of excoriated papules over trunk and extremities. On histology, there is hyperkeratosis, acanthosis, spongiosis and superficial perivascular, periadnexal perifollicular infiltration of lymphocytes. CD4 counts less than 200 were found in majority of PPE cases indicating the need for initiating HAART therapy. Presence of perifollicular infiltrate of eosinophils is the characteristic feature of Eosinophilic folliculitis of HIV. Presence of neutrophilic infiltrate in the epidermis with dilated adnexal ducts was observed in Pityrosporum folliculitis. Hence, there is a need for histopathology examination to separate these closely related entities for precise diagnosis and proper management in HIV patients.

\section{Limitations}

Limitations of this study were small sample size, immunohistochemical markers could not be done due to resource limited settings. Further studies were needed to get the clear consensus on the aetiology of PPE.

\section{ACKNOWLEDGEMENTS}

We sincerely thank the Department of Pathology, RMC Kakinada and Medical officer, ART centre, GGH, Kakinada for their help in conducting the study.

\section{REFERENCES}

[1] Bason MM, Berger TG, Nesbitt LT. Pruritic papular eruption of HIV-disease. Int J Dermatol 1993;32(11):784-9.

[2] Fishek BK, Warner LC. Cutaneous manifestations of the acquired immunodeficiency syndrome. Int $\mathrm{J}$ Dermatol 1987;26(10):615-30.
[3] Pruritic papular eruption of HIV. Dermanet NZ, Bentallon Dermatology registrar, Auckland green Hospital Draft, 2007.

http://www.dermnetnz.org/viral/papulopruritic.hiv. html

[4] Gelfand JM, Rudikoff D. Evaluation and treatment of itching in HIV infected patients. Mt Sinai J Med 2001;68(4-5):298-308.

[5] Farsani TT, Kore S, Nadol P, et al. Etiology and risk factors associated with a pruritic papular eruption in people living with HIV in India. J Int AIDS Soc 2013;16(1):17325.

[6] Ramos H, Pagliari C, Takakura CF, et al. Pruritic papular eruption associated with HIVetiopathogenesis evaluated by clinical, immunohistochemical and ultrastructural analysis. J Dermatol 2005;32(7):549-56.

[7] Eisman S. Pruritic papular eruption in HIV. Dermatol Clin 2006;24(4):449-57. derm.theclinics.com.

[8] Singh H, Singh P, Tiwari P, et al. Dermatological manifestations in HIV infected patients at a tertiary care hospital in a tribal (Bastar) region of Chhattisgarh, India. Indian J Dermatol 2009;54(4):338-41.

[9] WHO statistics. 2005. www.hiv/aids.gov.in facts and figures.

[10] NACO guidelines, 2016. naco.gov.in HIV facts and figures.

[11] UNAIDS Epidemiological fact sheet on HIV/AIDS 2008 update: New Delhi, India.

[12] AjithKumar K, George S, Babu PG. Abnormal insect bite reactions: a manifestation of immunosuppression of HIV infection? Indian J Dermatol Venereol Leprol 2001;67(2):72-4.

[13] Colebunders R, Mann JM, Francis H, et al. Generalized papular pruritic eruption in African patients with HIV infection. AIDS 1987;1(2):117-21.

[14] Havia 0, Jiminez-Aeosta F, Ceballos PL. Pruritic papular eruption of the acquired immunodeficiency syndrome: a clinic pathologic study. J Am Acad Dermatol 1991;24:231-5.

[15] Lakshmi SJ, Rao GR, Ramalakshmi, et al. Pruritic papular eruptions of HIV: a clinicopathologic and therapeutic study. Indian J Dermatol Venereol Leprol 2008;74(5):501-3.

[16] Resneck JS, van Beek M, Furmanski L, et al. Etiology of pruritic popular eruption with HIV infection in Uganda. JAMA 2004;292(21):2614-21.

[17] Castelnuovo B, Byakwaga H, Menten J, et al. Can response of a pruritic papular eruption to antiretroviral therapy be used as a clinical parameter to monitor virological outcome? AIDS 2008;22(2):26973.

[18] Pennys NS, Nayar JK, Bernstein H, et al. Chronic pruritic eruption in patients with acquired immunodeficiency syndrome associated with increased antibody titres to mosquito salivary gland antigens. J Am Acad Dermatol 1989;21(2):421-5.

[19] Boonchai W, Laohasrisakul R, Manonukul J, et al. Pruritic papular eruption in HIV seropositive patients: a cutaneous marker for immunosuppression. Int I Dermatol 1999;38(5):348-50. 
[20] Parker SR, Parker DC, McCall CO. Eosinophilic folliculitis in HIV infected women: case series and review. Am J Clin Dermatol 2006;7(3):193-200.

[21] Pityrosporum (Malassezia) folliculitis updated on July 10th 2017. emedicine.medscape.com

[22] Levers text book of histopathology. 9th edn. UK: Lippincott Raven 2004: p. 182.

[23] Aires JM, Rosatelli JB, de Castro FJF, et al. Cytokines in the pruritic popular eruption of HIV. Int J Dermatol 2000;39(12):903-6.
[24] Wichai. Cutaneous manifestations in HIV positive patients. South East Asian J Trop Med Public Health 2001:32(1).

[25] Wiwanitkit V. Prevalence of dermatological disorders in Thai HIV infected patients correlated with different CD4 lymphocyte count statuses: a note on 120 cases. Int J Dermatol 2004;43(4):265-8.

[26] Sanchez M, Fotiades J, Soter NA, et al. The characterization of HIV associated papular eruptions. Int Conf AIDS 1993;9:447. 